\title{
Association of genetic polymorphisms in the telomerase reverse transcriptase gene with prostate cancer aggressiveness
}

\author{
DAPENG WU ${ }^{1 *}$, HONGJIE YU²*, JIELIN SUN ${ }^{3}$, JUN QI $^{1}$, QIANG LIU ${ }^{1}$, RUIPENG LI $^{1}$, \\ SIQUN LILY ZHENG ${ }^{3}$, JIANFENG XU ${ }^{2,4}$ and JIAN KANG ${ }^{1}$

\begin{abstract}
${ }^{1}$ Department of Urology, Xinhua Hospital, Shanghai Jiaotong University School of Medicine, Shanghai 200092;
${ }^{3}$ Center for Cancer Genomics, Wake Forest University School of Medicine, Winston-Salem, NC 27157, USA;

${ }^{4}$ Fudan Institute of Urology, Huashan Hospital, Fudan University, Shanghai 200040, P.R. China
\end{abstract} \\ ${ }^{2}$ State Key Laboratory of Genetic Engineering, School of Life Sciences, Fudan University, Shanghai 200433, P.R. China;
}

Received May 9, 2014; Accepted January 30, 2015

DOI: $10.3892 / \mathrm{mmr} .2015 .3410$

\begin{abstract}
Telomerase reverse transcriptase (TERT), encoded by the TERT gene, is an essential component of telomerase, essential for the maintenance of telomere DNA length, chromosomal stability and cellular immortality. The aim of the present study was to evaluate the association between common genetic variations across the TERT gene region and prostate cancer (PCa) aggressiveness in a Chinese population. A total of 12 TERT tagging single-nucleotide polymorphisms (SNPs) were genotyped on the Sequenom Mass-ARRAY iPLEX ${ }^{\circledast}$ platform in a case-case study with 1,210 Chinese patients with $\mathrm{PCa}$. Unconditional logistic regression was used to investigate the association of genotypes with PCa aggressiveness, Gleason grade and risk of developing early-onset PCa. It was observed that the $\mathrm{C}$ allele of the TERT intron 2 SNP (rs2736100) was significantly associated with reduced risk of $\mathrm{PCa}$ aggressiveness [odds ratio $(\mathrm{OR})=0.81 ; 95 \%$ confidence interval $(\mathrm{CI})$ : 0.66-0.99; $\mathrm{P}=0.037]$. This allele was also significantly correlated with a reduced risk of developing a tumor with a high Gleason score ( $>7$; OR=0.83; 95\% CI: 0.70-0.99; $\mathrm{P}=0.039)$. The $\mathrm{T}$ allele of the intron 4 SNP (rs10069690) was found to be significantly associated with a decreased risk for an aggressive form of PCa (OR=0.76; 95\% CI: 0.59-0.97; P=0.030). In addition, the A allele of rs 10078761 located at the $3^{\prime}$ end of the TERT gene exhibited a statistically significant association with the reduced risk of developing a higher grade disease $(\mathrm{OR}=0.48$; 95\% CI: 0.28-0.81; $\mathrm{P}=0.006)$. However, no association between TERT polymorphisms and age at diagnosis was
\end{abstract}

Correspondence to: Dr Jian Kang, Department of Urology, Xinhua Hospital, Shanghai Jiaotong University School of Medicine, 1665 Kongjiang Road, Shanghai 200092, P.R. China

E-mail: kjjjjd@yahoo.com

*Contributed equally

Key words: prostate cancer, aggressiveness, telomerase reverse transcriptase, single nucleotide polymorphism, Chinese observed in the present study. The present findings demonstrated for the first time, to the best of our knowledge, that genetic variations across the TERT gene are associated with $\mathrm{PCa}$ aggressiveness in a Chinese Han population.

\section{Introduction}

Telomerase is a ribonucleoprotein complex, which catalyzes the de novo addition of TTAGGG nucleotide repeat sequences to prevent telomere shortening at the distal ends of eukaryotic chromosomes. While telomerase may maintain chromosomal integrity and stability during division of actively dividing cells, it also enables cell proliferation, making it one of the primary factors leading to carcinogenesis. Telomerase activation has been detected in the vast majority of human carcinomas and in vitro immortalized cells with no detectable expression in normal stable human somatic cells $(1,2)$. In light of the characteristics above, human telomerase is one of the most promising tumor markers and a potentially highly specific molecular target for therapeutic interventions (3). Among several protein components of human telomerase, human telomerase reverse transcriptase (hTERT), as a catalytic subunit of the telomerase enzyme complex, has been observed to be the key determinant of enzymatic activity in human telomerase (4). By synthesizing multiple tandem repeats of DNA (namely telomeric DNA), hTERT, encoded by the TERT gene, compensates for the erosion of DNA ends during replication and provides docking sites for telomeric proteins that bind specifically to the ends of chromosomes (5). Mutations in the TERT gene regions may affect telomerase activity and it was observed in several studies, which performed TERT single-nucleotide polymorphism (SNP) analysis, that this gene had a role in susceptibility to tumorigenesis in multiple types of cancer $(2,6,7)$.

Prostate cancer (PCa) is a significant health problem for older males, with an estimated 233,000 novel cases and 29,480 cancer-associated fatalities expected in 2014 in the United States alone (8). The widespread use of prostate-specific antigen (PSA) screening, which may result in a decrease in PCa mortality, has led to the overdetection, overtreatment and increasing costs of this highly heterogeneous disease with diverse clinical outcomes $(9,10)$. Side effects due to 
overtreatment and their negative impact on the patient's quality of life justify the importance of sparing patients from unnecessary treatment and the requirement for specific markers indicating disease prognosis (11). Although factors such as the Gleason score and tumor stage are used to assess prognosis, there remains a requirement for improved biomarkers to distinguish between PCa cases that may likely recur, progress rapidly and be life-threatening versus those that may not have a substantial impact on mortality (12).

In two recent studies it was suggested that quantification of $T E R T$ expression may be a valuable non-invasive marker for discriminating between localized and locally advanced PCa, as well as a useful tool for the early prediction of biochemical recurrence of PCa $(9,13)$. Another study using immunohistochemistry demonstrated that the immunoreactivity of hTERT may be used as a molecular marker for high-grade prostate cancer (14). However, the role of TERT genetic variations in PCa progression remains to be elucidated. An aim of the present study was to therefore clarify the association between TERT locus polymorphisms and PCa aggressiveness in an in-patient Chinese patient cohort. To the best of our knowledge, the present study was the first to evaluate the effect of these variations on PCa severity.

\section{Materials and methods}

Study population. Between February 2010 and April 2013, PCa patients who were between 34 and 97 years old at the time of diagnosis were recruited from the Departments of Urology at Xinhua Hospital (School of Medicine, Shanghai Jiao Tong University) and Huashan Hospital (Fudan University) in Shanghai, China. The study protocol was approved by the Science and Technology Commission of Shanghai Municipality and institutional review boards of Xinhua Hospital and Huashan Hospital. All subjects received a detailed description of the study protocol and provided informed consent. All eligible subjects included in the present study were of Chinese Han ancestry. The general eligibility criteria were: i) Newly diagnosed PCa cases with histologically confirmed disease; ii) ability of the patient to comprehend informed consent and iii) no previous diagnosis of cancer. The exclusion criteria included patients with chronic inflammatory conditions, infections within the past six weeks and autoimmune diseases. A total of 1,210 individuals who met the criteria were selected for genotyping. The age at diagnosis was calculated from the date of the first positive biopsy and the serum PSA levels (defined as the most recent PSA value within 1 year prior to the diagnosis date) were obtained from a medical record review.

Histopathological grading of biopsies and radical prostatectomy specimens were performed according to the Gleason scoring system (15). Clinical and pathological stages were determined according to the 2010 American Joint Committee on Cancer (AJCC) tumor, nodes and metastasis (TNM) classification system. For Gleason scores and tumor stage information, values from prostatectomy were used whenever available; otherwise, biopsy values were used. The D'Amico risk classification criteria were used to predict the prognosis of patients with localized $\mathrm{PCa}(16)$, and patients in the present study were grouped as low-, moderate- or high-risk for clinical recurrence and rapid progression following primary therapy for PCa. Patients diagnosed with N1 (involvement of regional lymph nodes) or M1 (distant metastasis) PCa were included in the high-risk class. In addition, due to comparably small numbers of low- and moderate-risk PCa cases, they were combined into a single non-aggressive group. Thus, a total of 911 high-risk (aggressive) and 259 low/moderate-risk (non-aggressive) PCa cases were included in the present study. The remaining 40 patients could not be classified due to absent phenotypic data.

Selection of SNPs. A tagging approach was employed to perform a comprehensive evaluation of genetic variants across the TERT gene for their association with PCa aggressiveness. To include the probable regulatory regions of the TERT gene, the upstream of the initial gene region was extended for $10 \mathrm{~kb}$ and the downstream for $10 \mathrm{~kb}$ and thus the peak signals were at $61.9 \mathrm{~kb}$ (chr5:1, 296, 287-1, 358, 162, dbSNP b126). Subsequently, a greedy algorithm was used, based on the $\mathrm{r}^{2}$ statistic to identify tagging SNPs (tSNPs) using the Haploview program version 4.2 from the Broad Institute (http://www.broadinstitute.org/mpg/haploview) according to the HapMap database (http://www.hapmap.org/, HapMap Data Rel 24/phaseII Nov08, on NCBI B36 assembly, dbSNP b126; population: $\mathrm{CHB}+\mathrm{JPT}$ ) on the basis of pairwise linkage disequilibrium (LD) $\mathrm{r}^{2}$ threshold of 0.8 , Hardy-Weinberg Equilibrium $(\mathrm{HWE})=0.05$, minor-allele frequency $=0.01$ and call rate $=95 \%$. As a result, two SNPs (rs12513872 and rs6554691) in Block1 and one SNP (rs2736118) in Block2 were excluded from the panel due to $\operatorname{LD}\left(\mathrm{r}^{2}>0.8\right)$. In addition, one SNP (rs4246742) was eliminated from the analysis due to difficulty in designing primers for the genotyping assay. Finally, a total of 12 SNPs which met the above criteria were analyzed for the present study (Fig. 1).

Genotyping. Blood samples were collected from all study subjects and DNA was extracted using a whole blood genomic DNA extraction kit (Qiagen, Chatsworth, CA, USA) and then diluted to the concentration of $15-20 \mathrm{ng} / \mathrm{l}$ through the use of an ultraviolet spectrophotometer (Nanodrop 8000; Thermo Fisher Scientific, Waltham, MA, USA). Amplification of polymorphism flanking fragments and single base extension were conducted by the polymerase chain reaction (GeneAmp PCR Thermocycle Instrument 2720 and ABI PCR Thermocycle Instrument 9700; Applied Biosystems, Inc., Carlsbad, CA, USA). The 12 SNPs were genotyped for all subjects using a MassARRAY iPLEX system (Sequenom, Inc., San Diego, CA, USA) at Fudan University in Shanghai, China. A total of two duplicates and two water samples were included in each 96-well plate as polymerase chain reaction (PCR)-negative controls. All assays were performed by technicians in a blinded manner. The average concordance rate between samples was $>99 \%$ among the duplicated quality control samples and the genotyping missing rate was $2.5 \%$ for all samples.

Statistical analysis. The genotype distribution for each tSNP was assessed for the HWE using Pearson's goodness-of-fit. To investigate the association of genotypes with PCa aggressiveness (aggressive PCa vs. non-aggressive PCa), the Gleason score (>7 vs. $\leq 7)$ and the risk of developing early-onset PCa ( $\leq 60$ vs. $>60$ ), the odds ratios (ORs), $95 \%$ confidence intervals 


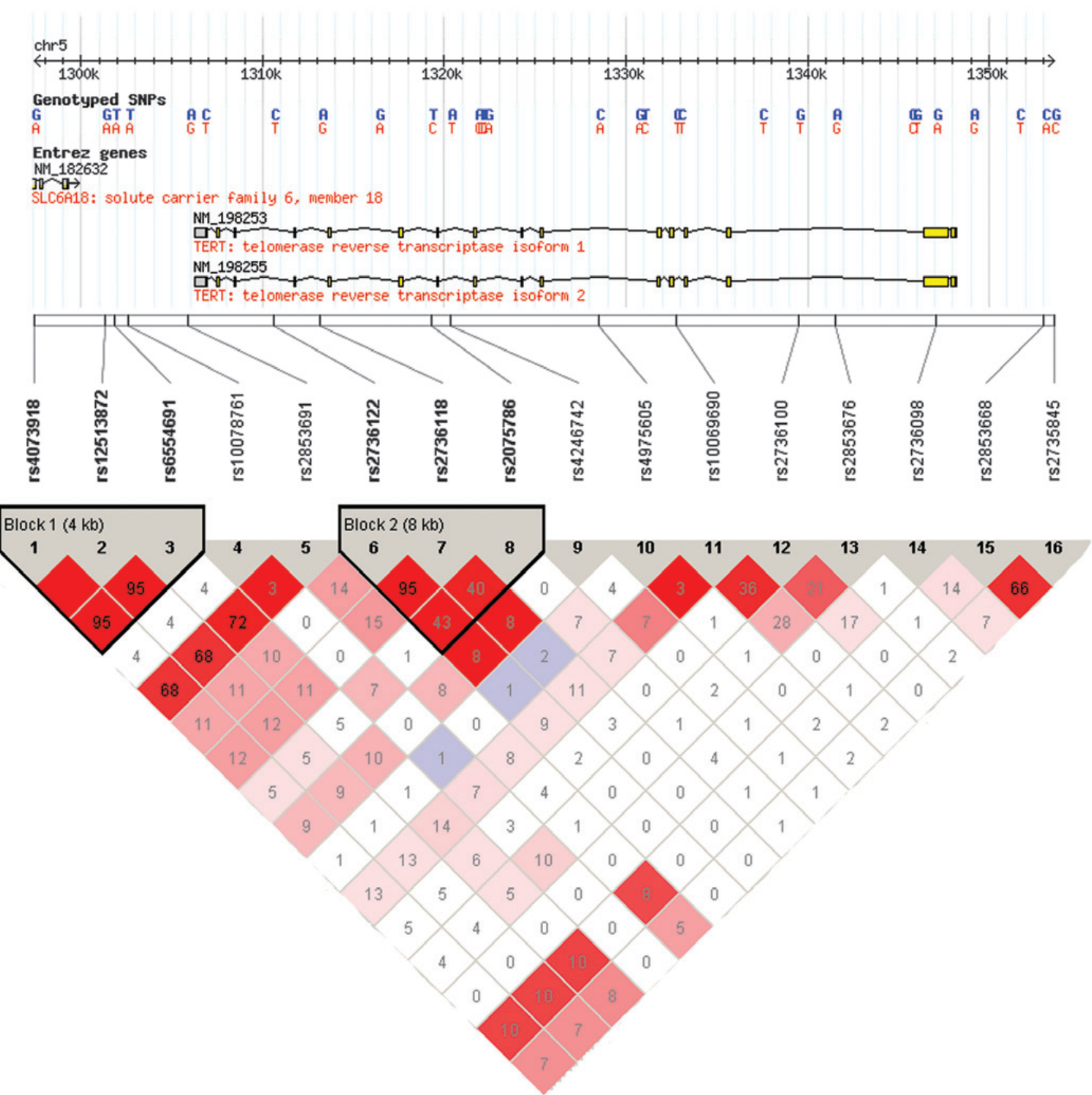

Figure 1. SNPs in the TERT gene locus and their linkage disequilibrium status. The panel demonstrates the patterns of linkage disequilibrium of the TERT gene locus based on HapMap phase 2 data on the CHB population (Release \#24, NCBI build36) using Haploview software. The upper panel demonstrates the TERT gene locus diagram and tagging SNPs created by Haploview 4.2. The lower panel demonstrates the linkage disequilibrium status between selected SNPs and the numbers shown in the plot were the values for pairwise $\mathrm{r}^{2}$. SNP, single-nucleotide polymorphism; CHB, Chinese Han population in Beijing; TERT, telomerase reverse transcriptase.

(CIs) as well as corresponding P-values were calculated using unconditional logistic regression with adjustment for age (as a continuous variable). Each tSNP was analyzed using additive, dominant, recessive and co-dominant models, respectively. All data were analyzed using PLINK 1.07 software (17). P-values were two-tailed and $\mathrm{P}<0.05$ was considered to indicate a statistically significant difference.

\section{Results}

Patient characteristics and clinical features. The distribution of demographic characteristics and clinical features of 1,210 PCa patients who were successfully genotyped are presented in Table I. The median age at diagnosis for these patients was 72 years (range, 34-97 years). A total of 1,165 patients had available PSA levels at diagnosis, with a median PSA of $6 \mathrm{ng} / \mathrm{ml}$ [Q1 (lower quartile), (Q3 upper quartile): 5, $11 \mathrm{ng} / \mathrm{ml}$. Among 1,171 patients with Gleason score information, 452 patients $(38.6 \%$ ) had Gleason scores $>7$, 400 patients $(34.2 \%)$ had scores of 7 , and 319 patients (27.2\%) had scores <7. Among patients who had AJCC clinical stage information available, 648 patients $(60.8 \%)$ had organ-confined tumors (T1/T2) and 418 patients $(39.2 \%)$ had extraprostatic (T3/T4) disease. In addition, 291 patients 
Table I. Patient characteristics and clinical features.

\begin{tabular}{|c|c|c|c|}
\hline \multirow[b]{2}{*}{ Patient characteristic } & \multicolumn{3}{|c|}{ Cases, n (\%) } \\
\hline & High risk $(\mathrm{n}=911)$ & $\begin{array}{l}\text { Low/moderate } \\
\text { risk }(n=259)\end{array}$ & All cases $(n=1,210)^{a}$ \\
\hline Patients with available age, $n$ & 906 & 258 & 1,204 \\
\hline Age at diagnosis (years; mean $\pm \mathrm{SD}$ ) & $71.36 \pm 8.26$ & $71.21 \pm 7.37$ & $71.32 \pm 8.06$ \\
\hline$\leq 60, \mathrm{n}(\%)$ & $89(9.8)$ & $19(7.4)$ & $113(9.39)$ \\
\hline$>60, \mathrm{n}(\%)$ & $817(90.2)$ & $239(92.6)$ & $1,091(90.61)$ \\
\hline Patients with PSA levels at diagnosis, n (\%) & $890(76.4)$ & $256(22.0)$ & 1,165 \\
\hline $0-4$ & $28(2.4)$ & $22(1.9)$ & $51(4.38)$ \\
\hline $4.01-10$ & $61(5.2)$ & $90(7.7)$ & $156(13.4)$ \\
\hline $10.01-20$ & $116(10.0)$ & $144(12.4)$ & $273(23.4)$ \\
\hline$>20$ & $685(58.8)$ & 0 & $685(58.8)$ \\
\hline Patients with Gleason score, n (\%) & $883(75.4)$ & $248(21.2)$ & 1,171 \\
\hline$>7$ & $452(38.6)$ & 0 & $452(38.6)$ \\
\hline 7 & $287(24.5)$ & $98(8.4)$ & $400(34.2)$ \\
\hline$<7$ & $144(12.3)$ & $150(12.8)$ & $319(27.2)$ \\
\hline \multicolumn{4}{|l|}{ Pathological tumor stage, $\mathrm{n}(\%)^{\mathrm{b}}$} \\
\hline T stage & $808(75.8)$ & $258(24.2)$ & 1,066 \\
\hline $\mathrm{T} 1-\mathrm{T} 2(\%)$ & $390(36.6)$ & $258(24.2)$ & $648(60.8)$ \\
\hline T3-T4 (\%) & $418(39.2)$ & 0 & $418(39.2)$ \\
\hline N stage & 708 (73.9) & $250(26.1)$ & 958 \\
\hline N0 & $417(43.5)$ & $250(26.1)$ & 667 (69.6) \\
\hline N1 & $291(30.4)$ & 0 & $291(30.4)$ \\
\hline M stage & $808(76.2)$ & $253(23.8)$ & 1,061 \\
\hline M0 & $478(45.1)$ & $253(23.8)$ & $731(68.9)$ \\
\hline M1 & $330(31.1)$ & 0 & $330(31.1)$ \\
\hline
\end{tabular}

${ }^{a} 40$ patients could not be classified as having aggressive or nonaggressive disease due to missing phenotypes. ${ }^{\mathrm{b}} \mathrm{T} 1-\mathrm{T} 2$ indicating T1-T2, N0 or Nx, M0 or Mx; T3-T4 indicating T3-T4, N0 or Nx, M0 or Mx; N1 indicating T1-T4 or Tx, N1, M0 or Mx; M1 indicating T1-T4 or Tx, N0 or $\mathrm{Nx}, \mathrm{M} 1$ according to the American Joint Committee on Cancer staging. PSA, prostate-specific antigen.

(30.4\%) had lymphatic metastasis, while 330 patients $(31.1 \%)$ had distant metastasis. In total, sufficient information for the modified criteria of the D'Amico risk classification was available for 1,170 patients, of whom 911 (77.9\%) were high-risk and 259 (22.1\%) were low/moderate-risk.

Association of TERT $t$ SNPs with prostate cancer aggressiveness. All tSNPs were within the HWE (all P>0.05) and had a missing rate $<0.05$. Initially, the association of the tSNPs across the TERT gene with PCa aggressiveness was assessed (Table II). A total of two SNPs (rs2736100 and rs10069690) were significantly associated with aggressiveness, assuming an additive effect $(\mathrm{P}=0.037$ and 0.030 , respectively). Individuals that carried the homozygous $\mathrm{C}$ allele of the TERT intron 2 SNP (rs2736100) had an OR of 0.81 (95\% CI: 0.66-0.99), indicating reduced $\mathrm{PCa}$ aggressiveness in comparison to those that carried the A allele. Males that carried the homozygous T allele of the TERT intron 4 SNP (rs10069690) had an OR of 0.76 (95\% CI: 0.59-0.97), indicating reduced $\mathrm{PCa}$ aggressiveness in comparison to those that carried the $\mathrm{C}$ allele. Individuals that carried the TC and TT genotypes of rs10069690 had a further reduced risk of developing an aggressive form of $\mathrm{PCa}(\mathrm{OR}=0.69,95 \%$ CI: 0.52-0.93), compared with males that carried the CC genotype, assuming a dominant model. Subsequently, it was further evaluated whether these two SNPs conferred an independent effect on PCa aggressiveness. A multivariate logistic regression, which included SNPs and age in the model, revealed non-significant associations for the two SNPs $(\mathrm{P}=0.203$ and $\mathrm{P}=0.188$ for $\mathrm{rs} 2736100$ and $\mathrm{rs} 10069690$, respectively), which indicated a non-independent effect between these two SNPs $\left(\mathrm{r}^{2}=0.36 ; \mathrm{D}^{\prime}=0.91\right)$.

Association of TERT tSNPs with Gleason score. The association between TERT tSNPs and Gleason score was estimated (Table III). Compared with the A allele, the C allele of rs2736100 conferred a reduced risk of developing high-grade $\mathrm{PCa}$ with an OR of 0.83 (95\% CI: 0.61-0.99; P=0.039). Rs10069690 was not significantly associated with high-grade $\mathrm{PCa}$. In addition, no rare homozygotes for rs10078761, which is located $3^{\prime}$ of the TERT gene, were observed in the present study cohort. The TT, AT and AA genotype distributions for rs10078761 were 1,105, 79 and 0 , respectively, among the 1,184 samples that had genotyping 


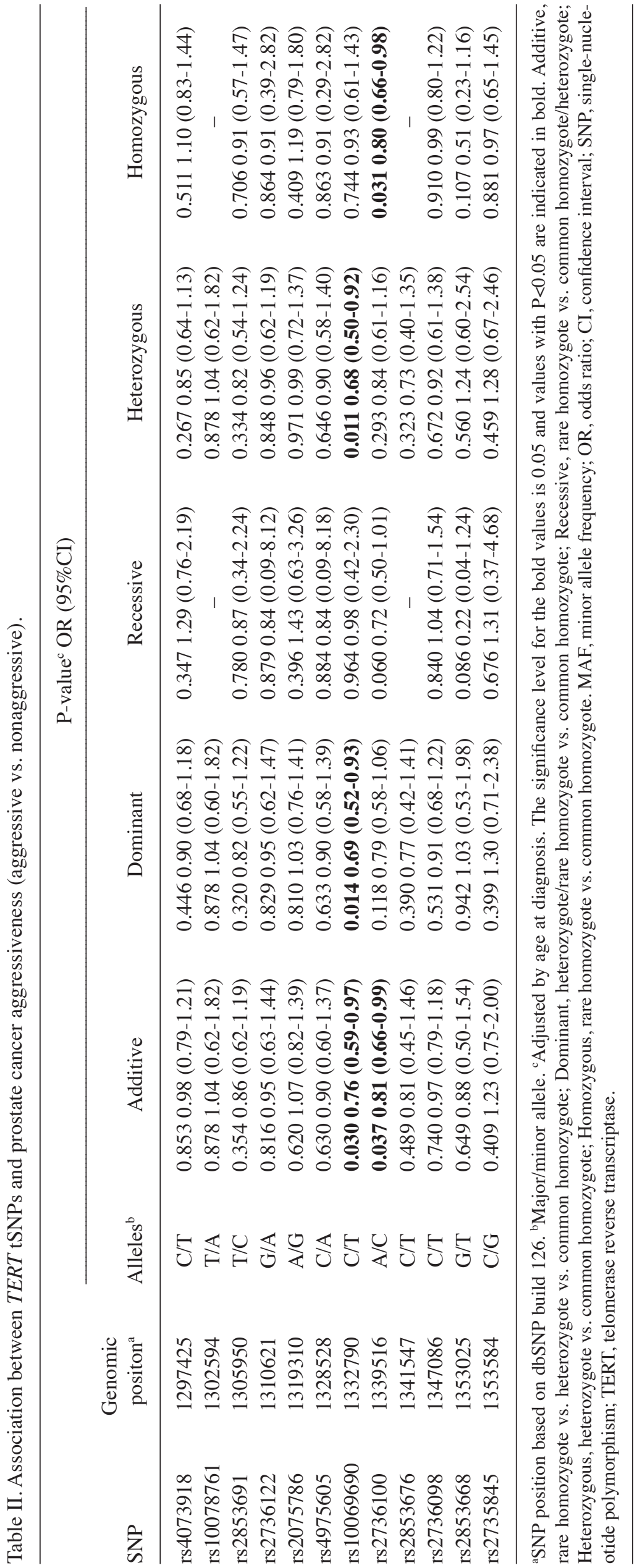




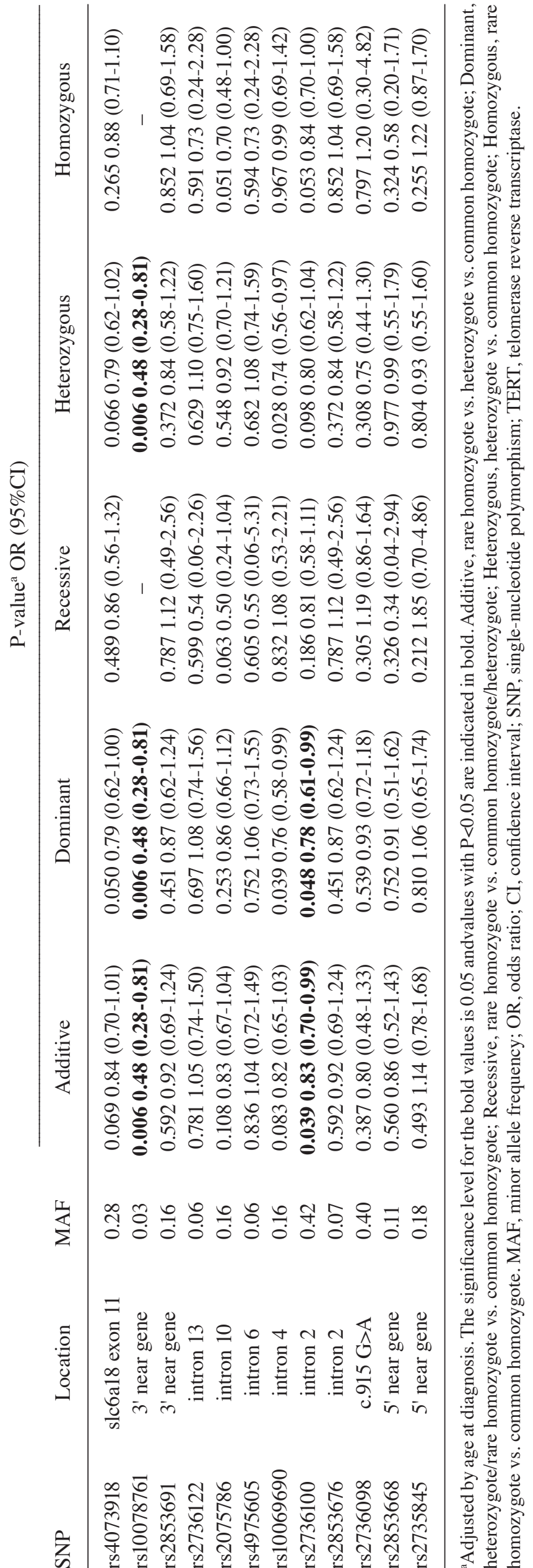


data available. In addition, this SNP was significantly associated with high-grade tumors. Individuals that carried the A allele of rs10078761 had a significantly decreased risk for developing high-grade $\mathrm{PCa}(\mathrm{OR}=0.48$; 95\% CI: 0.28-0.81; $\mathrm{P}=0.006)$.

In addition, no significant association between TERT tSNPs and the risk of developing an early-onset $\mathrm{PCa}$ (age $\leq 60$ at diagnosis) was observed in the present study (data not shown).

\section{Discussion}

The incidence of $\mathrm{PCa}$ in China has risen rapidly in recent years. It is well established that males diagnosed with low or moderate-risk PCa, based on the D'Amico classification criteria, are less likely to experience progression to metastasis. Due to the growing popularity of active surveillance and minimally invasive therapies, it is extremely important to identify which cases are to follow a more indolent course, by contrast to those that require aggressive treatment to improve prognosis. Such knowledge would enable clinicians to optimize the quality of life of patients who are at a lower risk for disease aggressiveness and thus may be spared unnecessary therapy and direct more radical therapies to those with the greatest requirement. Genetic factors offer a potentially promising avenue for further clarification of PCa aggressiveness (18). Accordingly, there is an urgent requirement for molecular biomarkers enabling improved prediction of $\mathrm{PCa}$ behavior and identification of patients with $\mathrm{PCa}$ who harbor potentially aggressive disease and those that do not. Due to the important role of the TERT gene in PCa progression, as previously reported $(9,12,13)$, it was hypothesized that TERT SNPs may be associated with PCa aggressiveness. Therefore, the genetic variations across the TERT gene were systematically evaluated for their impact on PCa severity in a Chinese Han population of 1,210 cases in the present study.

The TERT gene is located on the short (p) arm of chromosome 5 at position 15.33 and consists of 16 exons and 15 introns spanning $35 \mathrm{~kb}$ of genomic DNA (19). TERT, as the reverse transcriptase component of telomerase, was found to be rate-limiting for telomerase activity and a tight regulator of telomerase activity at the transcriptional and post-translational levels (4,20). March-Villalba et al (9) used quantitative RT-PCR to determine plasma hTERT mRNA levels in patients with localized and locally advanced PCa, respectively. The authors observed that patients with locally advanced disease had significantly higher plasma hTERT mRNA expression than those with localized disease. Sabaliauskaite et al (13) confirmed that TERT-positive PCa cases had elevated levels of ETS-related gene $(E R G)$, of which the fusion with transmembrane protease, serine 2 was not only a significant event of prostate tissue malignization but also associated with more aggressive disease and worse prognosis (21), suggesting a possible association between aberrant expression of $E R G$ and reactivation of TERT in prostate tumors. In addition, Iczkowski et al (14) used immunohistochemistry to detect the association between a polyclonal antibody to TERT and the Gleason score of cancer, where it was demonstrated that nuclear anti-TERT reactivity was restricted to high-grade carcinoma (Gleason primary pattern $\geq 4$ ). The above studies all suggested a correlation between TERT and PCa aggressiveness.
At present, introns are becoming increasingly recognized as having significant roles in gene regulation, including containing silencer or enhancer elements, alternative splicing and exon shuffling (22). SNP rs2736100, which is situated in intron 2 of TERT, lies in a putative regulatory region according to the Evolutionary and Sequence Pattern Extraction through Reduced Representation score (23). In the present study, it was identified that rs2736100 was associated with decreased PCa aggressiveness and degree of differentiation (i.e. the major $\mathrm{A}$ allele of rs2736100 was associated with a poorer degree of differentiation of prostate cancer compared with the minor $\mathrm{C}$ allele). A recent functional study demonstrated that the mutational CC genotype of this SNP was associated with lower telomerase activity and longer telomere length (TL) compared with the wild-type, as elucidated using a TRAPeze telomerase detection kit and quantitative RT-PCR-based assays, respectively (24). The lower telomerase activity observed in the aforementioned study is consistent with the results of the present study, indicating that the $\mathrm{CC}$ genotype is associated with suppression of PCa progression. As for the TL, a previous study demonstrated the inverse association between TL and cancer incidence and mortality (25). Telomere shortening may cause telomere dysfunction, ongoing chromosomal instability and ultimately lead to an increased risk of cancer development (26).

Although a population-based case-control study failed to observe a statistically significant association between leukocyte TL and PCa risk using quantitative PCR (27), two meta-analyses revealed that shorter telomeres were significantly associated with an increased overall risk of cancer compared with longer telomeres (28). This may explain the present result in which the mutational CC genotype of SNP rs2736100, which is associated with longer TL, may reduce the risk of developing a more aggressive form of PCa. In addition, rs10069690, which is mapped to intron 4 of TERT, has been observed to increase the risk of developing ER-negative breast and ovarian cancer and also increase the risk for those carrying breast cancer 1 mutations; however, this occurs independently of altered TL. The present study demonstrated that the minor $\mathrm{T}$ allele (minor allele in Caucasian and Chinese populations) of rs10069690 was associated with longer TL, as with the SNP rs2736100, which indicated that the T allele was expected to inhibit the cancer development, while the evidence that it increased the risk of cancer was to the contrary. Such notable contradictions reflect the complexity of associations among genetic variations, telomere structures and clinical phenotypes. Another study demonstrated that the non-T allele of rs10069690 may increase the risk of development and metastasis in primary hepatocellular carcinoma in Chinese individuals (29). The different results of the two studies may be reflective of the different roles genetic variations have between tumorigenesis and tumor prognosis, and it may also reflect the tumor-specific effect and genetic heterogeneity effect among different ethnic populations with regards to cancer risk. In addition, the present study observed specific residual LD even when the tSNPs approach was used and this revealed the potential disadvantage of this approach. Thus, due to the LD between these two intron region tSNPs (rs2736100 and rs10069690), it was very difficult to separate their own effect in the genetic association study. 
Furthermore, to the best of our knowledge, no previous studies have reported the SNP rs10078761 which is located 3' of the TERT gene and none of the mutational homozygotes with the AA genotype of this SNP were detected in these subjects. Possible explanations for this result may be that the individuals carrying the AA genotype may be less likely to develop PCa; these individuals may not be viable beyond the embryonic period, or this may be attributed to the genotyping failure of the remaining 26 samples in the study population. This notable finding requires further comprehensive investigation to examine this intergenic variation in the future.

There are several limitations in the present study that should be discussed. Firstly, although the established D'Amico classification criteria has been generally accepted to predict the prognosis of PCa patients, PCa aggressiveness using this criteria may be affected by healthcare practices, including screening time and the frequency of physical examination. For instance, frequently examined individuals are more likely to be diagnosed with PCa at lower PSA levels and earlier tumor stages and thus more are classified as having a less aggressive disease. However, the course of the disease in a number of these individuals may progress very rapidly, which is only evident following subsequent clinical observations. Thus, there is the probability of misclassification of PCa aggressiveness in the present study. The ongoing collection of clinicopathological variables, including biochemical recurrence, clinical metastases and cancer-specific mortality for study subjects appears necessary for more accurate classification in the future (30). In addition, none of the observed associations may survive when the most stringent criteria to correct for multiple assessment (the Bonferroni correction) is taken into account, in which the corrected $\alpha$-value would be 0.0042 . Finally, this analysis of genotype profiling should be coupled with complementary studies aimed at setting a complete molecular signature of individuals, including epigenetic modifications and gene expression profiles in RNA and protein levels. Such a combination may be more precise in the comprehensive classification of disease severity than the current systems.

In conclusion, the present results indicated that genetic polymorphisms in the TERT gene are associated with PCa aggressiveness in a Chinese Han population. This finding provides evidence that TERT gene variations may be involved in PCa development, progression and metastasis, and may be used as a prognostic indicator. If further confirmed, these identified genetic variations may assist to clarify which carcinomas are more likely to progress rapidly and require more intensive treatment versus those that may not have a severe impact on mortality. Further validation in a larger set of $\mathrm{PCa}$ samples and subsequent functional studies of TERT polymorphisms are required to further evaluate the present findings.

\section{Acknowledgements}

The present study was funded by the Science and Technology Commission of Shanghai Municipality (grant no. 11ZR1424100) and partly supported by the Key Project of the National Natural Science Foundation of China (grant no. 81130047). The authors would like to thank all the study subjects who were involved in the present study for their time, effort and cooperation. The authors would also like to thank all staff members in the Department of Urology at Xinhua Hospital and Huashan Hospital for their cooperation during data collection.

\section{References}

1. Shay J and Bacchetti S: A survey of telomerase activity in human cancer. Eur J Cancer 33: 787-791, 1997.

2. Mocellin S, Verdi D, Pooley KA, et al: Telomerase reverse transcriptase locus polymorphisms and cancer risk: a field synopsis and meta-analysis. J Natl Cancer Instit 104: 840-854, 2012.

3. Harley CB: Telomerase and cancer therapeutics. Nat Rev Cancer 8: 167-179, 2008.

4. Horikawa I and Barrett JC: Transcriptional regulation of the telomerase hTERT gene as a target for cellular and viral oncogenic mechanisms. Carcinogenesis 24: 1167-1176, 2003.

5. Nandakumar J and Cech TR: Finding the end: recruitment of telomerase to telomeres. Nat Rev Mol Cell Biol 14: 69-82, 2013.

6. Baird DM: Variation at the TERT locus and predisposition for cancer. Expert Rev Mol Med 12: 1-21, 2010.

7. Rafnar T, Sulem P, Stacey SN, et al: Sequence variants at the TERT-CLPTM1 L locus associate with many cancer types. Nat Genet 41: 221-227, 2009.

8. Siegel R, Ma J, Zou Z and Jemal A: Cancer statistics, 2014. CA Cancer J Clin 64: 9-29, 2014.

9. March-Villalba JA, Martínez-Jabaloyas JM, Herrero MJ, Santamaría J, Aliño SF and Dasí F: Plasma hTERT mRNA discriminates between clinically localized and locally advanced disease and is a predictor of recurrence in prostate cancer patients. Expert Opin Biol Ther 12: 69-77, 2012.

10. Heijnsdijk E, Der Kinderen A, Wever E, Draisma G, Roobol M and De Koning H: Overdetection, overtreatment and costs in prostate-specific antigen screening for prostate cancer. Br J Cancer 101: 1833-1838, 2009.

11. Cheng I, Plummer SJ, Neslund-Dudas C, et al: Prostate cancer susceptibility variants confer increased risk of disease progression. Cancer Epidemiol Biomarkers Prev 19: 2124-2132, 2010.

12. FitzGerald LM, Kwon EM, Conomos MP, et al: Genome-wide association study identifies a genetic variant associated with risk for more aggressive prostate cancer. Cancer Epidemiol Biomarkers Prev 20: 1196-1203, 2011.

13. Sabaliauskaite R, Jarmalaite S, Petroska D, et al: Combined analysis of TMPRSS2-ERG and TERT for improved prognosis of biochemical recurrence in prostate cancer. Gene Chromosome Canc 51: 781-791, 2012.

14. Iczkowski KA, Pantazis CG, McGregor DH, Wu Y and Tawfik OW: Telomerase reverse transcriptase subunit immunoreactivity. Cancer 95: 2487-2493, 2002.

15. Gleason DF and Mellinger GT: Prediction of prognosis for prostatic adenocarcinoma by combined histological grading and clinical staging. J Urol 111: 58-64, 1974.

16. D'Amico AV, Whittington R, Malkowicz SB, et al: Biochemical outcome after radical prostatectomy, external beam radiation therapy, or interstitial radiation therapy for clinically localized prostate cancer. JAMA 280: 969-974, 1998.

17. Purcell S, Neale B, Todd-Brown K, et al: PLINK: a tool set for whole-genome association and population-based linkage analyses. Am J Hum Gen 81: 559-575, 2007.

18. Witte JS: Prostate cancer genomics: towards a new understanding. Nat Reviews Genet 10: 77-82, 2009.

19. Wick M, Zubov D and Hagen G: Genomic organization and promoter characterization of the gene encoding the human telomerase reverse transcriptase (hTERT). Gene 232: 97-106, 1999.

20. Wang S, Wu J, Hu L, et al: Common genetic variants in TERT contribute to risk of cervical cancer in a Chinese population. Mol Carcinog 51: E118-E122, 2012.

21. Kumar-Sinha C, Tomlins SA and Chinnaiyan AM: Recurrent gene fusions in prostate cancer. Nat Rev Cancer 8: 497-511, 2008.

22. Landi MT, Chatterjee N, Yu K, et al: A genome-wide association study of lung cancer identifies a region of chromosome 5p15 associated with risk for adenocarcinoma. Am J Hum Gene 85: 679-691, 2009.

23. Taylor J, Tyekucheva S, King DC, Hardison RC, Miller W and Chiaromonte F: ESPERR: learning strong and weak signals in genomic sequence alignments to identify functional elements. Genome Res 16: 1596-1604, 2006. 
24. Sheng X, Tong N, Tao G, et al: TERT polymorphisms modify the risk of acute lymphoblastic leukemia in Chinese children. Carcinogenesis 34: 228-235, 2013.

25. Willeit P, Willeit J, Mayr A, et al: Telomere length and risk of incident cancer and cancer mortality. JAMA 304: 69-75, 2010

26. Feldser DM, Hackett JA and Greider CW: Telomere dysfunction and the initiation of genome instability. Nat Rev Cancer 3: 623-627, 2003.

27. Mirabello L, Huang WY, Wong JY, et al: The association between leukocyte telomere length and cigarette smoking, dietary and physical variables and risk of prostate cancer. Aging cell 8: 405-413, 2009.
28. Ma H, Zhou Z, Wei S, et al: Shortened telomere length is associated with increased risk of cancer: a meta-analysis. PLoS One 6: e20466, 2011.

29. Dong J, Wang L, Tian Y, Guo Y and Liu H: hTERT single nucleotide polymorphism is associated with increased risks of hepatocellular carcinoma and tumor metastasis]. Nan Fang Yi Ke Da Xue Xue Bao 31: 49-52, 2011 (In Chinese).

30. Bensen JT, Xu Z, Smith GJ, Mohler JL, Fontham ET and Taylor JA: Genetic polymorphism and prostate cancer aggressiveness: A case-only study of 1,536 GWAS and candidate SNPs in African-Americans and European-Americans. Prostate 73: $11-22,2013$ 